\title{
Leishmania (Viannia) lainsoni: occurrence of intracellular promastigote forms in vivo and in vitro
}

\author{
José R Corrêa, Maurilio J Soares ${ }^{+}$
}

Laboratório de Biologia Celular de Microrganismos, Departamento de Ultra-estrutura e Biologia Celular, Instituto Oswaldo CruzFiocruz, Av. Brasil 4365, 21040-900 Rio de Janeiro, RJ, Brasil

Experimental chronic (45-day-old) skin lesion in hamster hind foot induced by Leishmania (Viannia) lainsoni infection showed the presence of promastigote forms in the tissue, inside parasitophorous vacuoles, as assessed by transmission electron microscopy. Experimental in vitro interaction (24 and 48 h) between Leishmania (V.) lainsoni and J774-G8 macrophage cells also demonstrated the same profile. This morphological aspect is unusual, since in this parasite genus only amastigote forms have been described as the resistant and obligate intracellular forms.

Key words: Leishmania - lesion - macrophage - ultrastructure

Parasitic protozoa belonging to the Leishmania genus are pathogens that alternate between two evolutive stages: a flagellate promastigote form, which lives as an extracellular parasite in the digestive tract of phlebotomine sand flies, and a non-motile amastigote form, which lives as an obligatory intracellular parasite of mammals. Macrophages are the preferred mammalian host cells, where amastigotes survive and multiply within membrane-bound compartments called parasitophorous vacuoles (Antoine et al. 1999). Leishmania (Viannia) lainsoni was first isolated from human single skin lesions in the state of Pará, at the Brazilian Amazon region (Silveira et al. 1987). Studies on this Leishmania species are of interest, as biological, biochemical, and molecular analysis revealed its divergent taxonomic position (reviewed in Corrêa et al. 2005a), as well as its atypical biological behavior in axenic culture (Corrêa et al. 2005b). Here we present an unusual characteristic of this parasite, which is the presence of the promastigote form in a 45-day-old hamster skin lesion. Similar promastigote forms could be also found inside parasitophorous vacuoles of J774-G8 macrophages in vitro, after 24 and $48 \mathrm{~h}$ of interaction. This record is remarkable within the Leishmania genus.

L. (V.) lainsoni (strain MHOM/BR/1981/6425) promastigotes were maintained axenically at $25^{\circ} \mathrm{C}$ by serial passages in LIT medium supplemented with $10 \%$ fetal calf serum (FCS). Stationary phase promastigotes $\left(1.0 \times 10^{6}\right.$ cells $/ \mathrm{ml}$ ) were rinsed three times in phosphate buffered saline, $\mathrm{pH} 7.2$ (PBS) and then about $100 \mu \mathrm{l}$ of this cell suspension were inoculated subcutaneously into the hind foot of a hamster (Mesocricetus auratus) and as a negative control another animal was inoculated with the same

\footnotetext{
Financial support: CNPq, Papes-IV/Fiocruz

${ }^{+}$Corresponding author: maurilio@ioc.fiocruz.br

Received 24 July 2006

Accepted 30 August 2006
}

volume of PBS. After 45 days the animals were sacrificed and a skin sample was obtained from the inoculation site and processed for conventional transmission electron microscopy (TEM). Briefly, the samples were fixed for $2 \mathrm{~h}$ with $2.5 \%$ glutaraldehyde in $0.1 \mathrm{M}$ cacodylate buffer $(\mathrm{pH}$ 7.2), washed in buffer and then post-fixed for $1 \mathrm{~h}$ with $1 \%$ osmium tetroxide $/ 0.8 \%$ potassium ferricyanide $/ 5 \mathrm{mM}$ calcium chloride in $0.1 \mathrm{M}$ cacodylate buffer ( $\mathrm{pH}$ 7.2). After dehydratation in acetone series, the samples were embedded in PolyBed-812 resin (Polysciences, Warrington, PA, US) and polymerized for $72 \mathrm{H}$ at $60^{\circ} \mathrm{C}$. Ultrathin sections were stained with uranyl acetate and lead citrate and then observed in a Zeiss (Oberkochen, Germany) EM10C transmission electron microscope.

Furthermore, a cell line of J774-G8 macrophage was maintained by plating in $25 \mathrm{~cm}^{3}$ culture flasks containing D-MEM medium (Sigma Chemical Co., St. Louis, MI, US) supplemented with $10 \% \mathrm{FCS}$ and incubated at $37^{\circ} \mathrm{C}$ in a $5 \% \mathrm{CO}_{2}$ atmosphere. Stationary phase promastigotes were resuspended in fresh D-MEM medium and then incubated at $34^{\circ} \mathrm{C}$ with about $3 \times 10^{6}$ macrophages, at a 5:1 (parasites:macrophage) ratio. After $2 \mathrm{~h}$ of interaction the infected cultures were rinsed with PBS to remove non-adhered parasites, and then further incubated at $37^{\circ} \mathrm{C}$ and $5 \% \mathrm{CO}_{2}$ atmosphere. The infected macrophages were maintained in cultures for 24 or $48 \mathrm{~h}$ and then harvested, fixed and processed as the biopsy material.

Analysis of the hamster 45-day-old skin lesion by TEM showed amastigote forms with the characteristic round shape, a centrally located nucleus, a bar-shaped kinetoplast, and large lipid inclusions (Fig. 1). However, unexpected intracellular promastigote forms were also found in the chronic lesion site, with the typical elongated body shape and a free flagellum (Fig. 1). Similarly, after $24 \mathrm{~h}$ of interaction between $L$. (V.) lainsoni and J774-G8 macrophages, promastigote forms could be found inside parasitophorous vacuoles located close to the host cell nucleus (Fig. 2). Occasionally, the promastigote form was observed inside parasitophorous vacuoles in cells that had been incubated for $48 \mathrm{~h}$ (Fig. 3).

Henriques and De Souza (2000), using L. (L.) amazonensis, have reported intravacuolar promastigote 

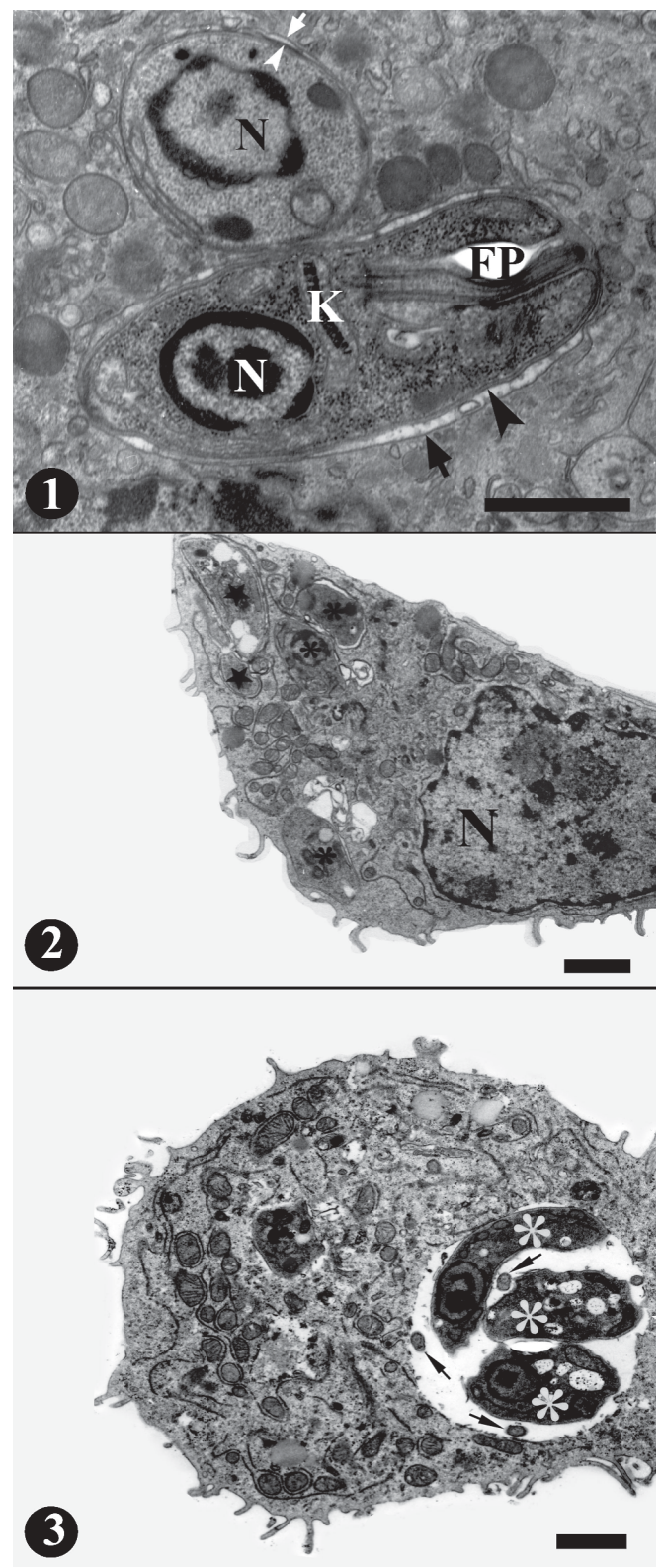

Fig. 1: transmission electron microscopy of a 45-day-old hamster skin lesion, showing amastigote and promastigote forms inside host cell vacuoles. Arrowheads: parasite membrane; arrows: parasitophorous vacuole membrane. N: nucleus; K: kinetoplast; FP: flagellar pocket. Fig. 2: infected J774-G8 macrophage after $24 \mathrm{~h}$ of interaction, containing intracellular amastigotes $(*)$ and promastigotes $(\star)$. N: nucleus. Fig. 3: infected J774-G8 macrophage after $48 \mathrm{~h}$ of interaction. Note the vacuole containing promastigotes (*) and flagella (black arrow). Bars $=1 \mu \mathrm{m}$. forms as a result of assays with short interaction periods (60 min). Unlike the former parasite, $L$. (V.) lainsoni exhibited promastigote forms in the hostile environment of macrophages both in vivo and in vitro after considerable longer periods.

We can speculate that such intracellular promastigote forms may arise several times during the mammal infection with $L$. $(V$.) lainsoni. The differentiation to promastigotes is usually associated to amastigotes released in the insect host after blood meal. While in the vertebrate host, amastigotes in the parasitophorous vacuole give rise to other amastigote forms. The interesting point in our results is that intravacuolar amastigotes may be giving rise to promastigote forms. At the moment we have no explanation for such phenomenon.

These unexpected characteristics of $L$. (V.) lainsoni deserve further investigation. Therefore, further biochemical and molecular analysis are ongoing with this $L$. (V.) lainsoni strain, in an attempt to obtain more knowledge on this unusual parasite differentiation.

\section{ACKNOWLEDGMENTS}

To Dr Fernando Silveira for kindly providing the Leishmania (Viannia) lainsoni strain MHOM/BR/1981/6425.

\section{REFERENCES}

Antoine JC, Lang T, Prina E, Courret N, Hellio R 1999. H-2M molecules, like MHC class II molecules, are targeted to parasitophorous vacuoles of Leishmania-infected macrophages and internalized by amastigotes of L. amazonensis and L. mexicana. J Cell Sci 112: 2559-2570.

Corrêa JR, Brazil RP, Soares MJ 2005a. Leishmania (Viannia) lainsoni (Kinetoplastida: Trypanosomatidae), a divergent Leishmania of the Viannia subgenus. Mem Inst Oswaldo Cruz 100: 587-592.

Corrêa JR, Santos SG, Araújo MS, Baptista C, Soares MJ, Brazil RP 2005b. Axenic promastigote forms of Leishmania (Viannia) lainsoni as an alternative source for Leishmania antigen production. J Parasitol 91: 551-556.

Henriques C, De Souza W 2000. Redistribution of plasmamembrane surface molecules during formation of the $L$. amazonensis-containing parasitophorous vacuole. Parasitol Res 86: 215-225.

Silveira FT, Shaw JJ, Braga RR, Ishikawa E 1987. Dermal leishmaniasis in the Amazon region of Brazil: Leishmania (Viannia) lainsoni sp.n., a new parasite from the state of Pará. Mem Inst Oswaldo Cruz 82: 289-292. 